University of Windsor

Scholarship at UWindsor

Winter 2009

\title{
A DEA-TOPSIS method for multiple criteria decision analysis in emergency management
}

Ye Chen

Kevin W. Li Dr.

University of Windsor

Haiyan Xu

Si-feng Liu

Follow this and additional works at: https://scholar.uwindsor.ca/odettepub

Part of the Business Commons

\section{Recommended Citation}

Chen, Ye; Li, Kevin W. Dr.; Xu, Haiyan; and Liu, Si-feng. (2009). A DEA-TOPSIS method for multiple criteria decision analysis in emergency management. Journal of Systems Science and Systems Engineering, 18 (4), 489-507.

https://scholar.uwindsor.ca/odettepub/67

This Article is brought to you for free and open access by the Odette School of Business at Scholarship at UWindsor. It has been accepted for inclusion in Odette School of Business Publications by an authorized administrator of Scholarship at UWindsor. For more information, please contact scholarship@uwindsor.ca. 


\title{
A DEA-TOPSIS method for multiple criteria decision analysis
}

\author{
Ye Chen ${ }^{a}$, Kevin W. Li ${ }^{b *}$, Haiyan $\mathrm{Xu}^{c}$, Si-feng Liu ${ }^{a}$, \\ ${ }^{a}$ College of Economics and Management, Nanjing University of Aeronautics and \\ Astronautics \\ Nanjing, 210016, China \\ ${ }^{b}$ Odette School of Business, University of Windsor, Windsor, ON, N9B 3P4, Canada \\ ${ }^{c}$ Department of Systems Design Engineering, University of Waterloo, Waterloo, ON, N2L \\ 3G1, Canada
}

\begin{abstract}
A hybrid approach of DEA (data envelopment analysis) and TOPSIS (technique for order performance (preference) by similarity to ideal solution) is proposed for multiple criteria decision analysis. Two DEA-based optimization models are constructed to facilitate identifying parameter information regarding criterion weights and quantifying qualitative criteria in TOPSIS. A numerical example is provided to demonstrate the proposed analysis procedure and carry out a comparative study. Analytic results show that these two models can provide relatively consistent results.
\end{abstract}

Key words: Multiple criteria decision analysis, multiple criteria ranking, DEA, TOPSIS, distance-based ranking

* Corresponding author. E-mail:kwli@uwindsor.ca (K. W. Li).

Preprint submitted to Journal of Systems Science and Systems Engineering18 February 2009 


\section{Introduction}

Multiple criteria decision analysis (MCDA) generally refers to decision aid tools that help decision-makers (DMs) in complex decision situations involving multiple criteria (objectives) arising from social, economic and environmental considerations $[15,17,23$, $26,27]$. The main idea of MCDA concentrates on decision analysis within a finite set of alternatives and offers techniques to assist individual DMs in making decisions by eliciting and aggregating their preferences. Two unique features of MCDA for handling decision problems are summarized below:

- Preference-based aggregation: To reach a conclusion, the performances of alternatives over all criteria have to be aggregated together. To achieve this more effectively, the aggregation in MCDA is conducted on DMs' preferences instead of the traditional cost-benefit analysis in which all criteria are converted to a monetised term as a common comparison ground. For example, in 1998, the Department of the Environment, Transport, and the Regions in the United Kingdom revealed an MCDA approach to appraising transport projects. This framework incorporates non-monetised considerations into the management planning process [11], thereby improving the project appraisal process.

- Ability to handle both quantitative and qualitative criteria: Both quantitative criteria that can be measured in numerical values objectively and qualitative criteria that can only be gauged subjectively are integrated together to generate comprehensive evaluations for all alternatives.

Roy [26] proposes that three MCDA problématiques (fundamental problems) are available for evaluating a set of alternatives, A, as per a DM's specific purpose.

- Choice. Choose the best alternative from A.

- Sorting. Sort the alternatives of A into relatively homogeneous groups, usually arranged in a preferential order.

- Ranking. Rank the alternatives of $\mathbf{A}$ from best to worst.

Among the above three types of decision analysis, ranking provides the most prolific information with a full preferential order for all alternatives, and the best alternative (choice) is identified as its by-product. A sorting problem can be addressed based on the generated ranking results by employing a logic group assignment procedure. For instance, the traditional ABC analysis, a well-known approach in inventory management [32], classifies items as per annual dollar usage (ADU) into three groups: the most important items (10-20\% of total items, but usually account for around $80 \%$ of ADU) are placed in group $\mathcal{A}$, demanding the greatest effort and attention from management, the least important items (40-50\% of total items, only account for around $10 \%$ of ADU) fall into group $\mathcal{C}$, where minimal effort is applied; other SKUs belong to the middle group $\mathcal{B}$. Recent efforts have been committed to this sorting problem to accommodate multiple criteria $[6,22]$. 
MCDA approaches are proposed from different schools of thinking, including multiattribute utility theory (MAUT) [23], outranking methods [26] and analytic hierarchy process (AHP) [27], to name a few. A state-of-the-art review of MCDA is provided in [15], summarizing many MCDA approaches catered for different decision scenarios.

The TOPSIS (technique for order performance (preference) by similarity to ideal solution) method was initially proposed in [17] to solve ranking problems. The basic idea of the TOPSIS is to first measure an alternative's distances to pre-defined ideal and anti-ideal points separately and, then, aggregate these two distances into an overall evaluation. As summarized in [24,30], TOPSIS possesses such attractive features as its clear and easily understandable geometric meaning, simultaneous considerations from both best and worst points of view, and convenient calculations and implementations. Therefore, different methods have been developed to refine the original TOPSIS idea such as those reported in $[1,2,4,5,19,30,31]$.

Data envelopment analysis (DEA) is an increasingly popular managerial decision tool that was initially proposed by Charnes et al. [3]. As a nonparametric method for estimating production frontiers, DEA measures relative performance of a set of producers or decision making units where the presence of multiple inputs and outputs makes comparisons difficult. A comprehensive survey of DEA research covering its 30 years of history (1978-2008) is presented in [14].

During the last thirty years, significant research has been conducted on DEA for both theoretical extensions and practical applications, including various DEA-based MCDA approaches. Among the early attempts, $[12,28]$ explored the utilization of cross-efficiency analysis in DEA for evaluating alternatives in MCDA, and Doyle [13] suggested that cross efficiency-based DEA analysis could be a "Multiattribute Choice (tool) for the Lazy Decision Maker: Let the Alternatives Decide!".

Stewart [33] compared ${ }_{\curlywedge}$ the goals of DEA and MCDA as "DEA arises from situations where the goal is to determine the productive efficiency of a system by comparing how well the system converts inputs into outputs, while MCDA models have arisen from the need to analyze a set of alternatives according to conflicting criteria". A methodological connection between MCDA and DEA is that if "all criteria in an MCDA problem can be classified as either benefit criteria (benefits or output) or cost criteria (costs or inputs), then DEA is equivalent to MCDA using additive linear value functions" [33].

Recently, several methods have been developed for MCDA by adding more preference information (constraints) into the DEA-like models. For example, Cook and Kress [10] proposes a DEA-based MCDA method to handle both cardinal and ordinal criteria; findings from [29] showed that "a modified DEA approach yields results very similar to those produced using SMART (simple multiattribute rating technique)"; a DEA-based index aggregation model has been developed in [7] for aggregating different country ranking indices; different DEA-based methods $[25,34]$ are proposed for weight deviation and aggregation in the AHP. 
Following this research direction, this paper puts forward a hybrid approach to MCDA that capitalizes on the unique features of DEA and TOPSIS as summarized below:

- A convenient way for determining criterion weights in TOPSIS

The identification of weights, the relative importance of criteria, constitutes an important task in MCDA. Various methods have been proposed for obtaining criteria weights in the literature. Different approaches have their merits and features. Søme ef them are summarized as follows: the AHP method and several other pair-wise comparison-based methods, such as geometric least squares method [18] have been widely used for weight deviation $[20]$. Swing weights [35] provides a convenient way for direct weighting. Indifference tradeoff weight as suggested by Keeney and Raiffa [23] is an indirect weighting technique by asking DMs to make tradeoffs and then deriving $_{\boldsymbol{\alpha}}$ the implied weights.

The TOPSIS method did not offer any technique to determine criterion weights as weights are pre-defined. The proposed DEA-TOPSIS method integrate $_{\lambda}$ weight devian ${ }_{\lambda}$ process into the TOPSIS method and automatically calculate the weight information by utilizing optimization programs under the philosophy of identifying individual alternatives' best possible overall performance. Such a process can provide a fair overall assessment of an alternative by maximizing its possibility of obtaining the best possible result and "has more of the right connotations of a democratic process" [12].

- A theoretically sound approach to qualitative criterion quantification in TOPSIS:

Usually there has been more preference uncertainty involving the determination of exact value for an ordinal criterion. For example, an interval datum may be more reasonable than an exact datum for $_{\mathbf{1}}$ representing preference over an ordinal criterion. Since the TOPSIS method did not provide any relevant process to handle the uncertainty in ordinal criteria, we adapted the method proposed by Cook and Kress [10] to solve this problem. The DEA-TOPSIS method provides a theoretically sound approach to quantifying qualitative criteria based on the aforesaid philosophy of individual performance optimization.

The remainder of the paper is organized as follows: Overviews of MCDA and TOPSIS are given in Section 2; Section 3 presents a hybrid method that integrates the DEA concept into TOPSIS; then, in Section 4, a numerical example adapted from [30] is used to demonstrate the proposed method, and, finally, some conclusions are given in Section 5 . 


\section{MCDA and TOPSIS methods}

\subsection{An overview of $M C D A$}

Analyzing an MCDA problem consists of three main steps: (1) Problem construction, where a list of all alternatives, criteria to assess alternatives, and the DM's objectives are identified. (2) Preference elicitation and aggregation, in which the DM's preferences within and across criteria are modeled and aggregated; (3) Implementation, in which the constructed preference model is applied to evaluate all alternatives, thereby solving the 'problématique' selected by the DM, as an aid to decision making.

Step (1) formulates a decision problem in the context of MCDA, identifying the set of alternatives, $\mathbf{A}=\left\{a^{1}, a^{2}, \cdots, a^{n}\right\}$ and the set of criteria, $\mathbf{C}=\left\{c_{1}, c_{2}, \cdots, c_{q}\right\}$. Step (1) also provides a direct physical measurement as the consequence of alternative $a^{i}$ on criterion $c_{j}$ for every $i=1, \ldots, n$ and $j=1, \ldots, q$, denoted by $m_{j}^{i}$, representing the $(i, j)$-entry of an $n \times q$ matrix, called the information (or performance) matrix. The format of this matrix is shown in Figure 1. Note that a consequence does not include preferential information.

Obviously, for quantitative criteria such as cost in dollars, objective measurements can be easily identified, while consequences for qualitative criteria such as the product quality may contain some ambiguity. It is a usual approach to assign a set of linguistic evaluations as consequences over qualitative criteria. More detailed explanations will be provided in Section 3.

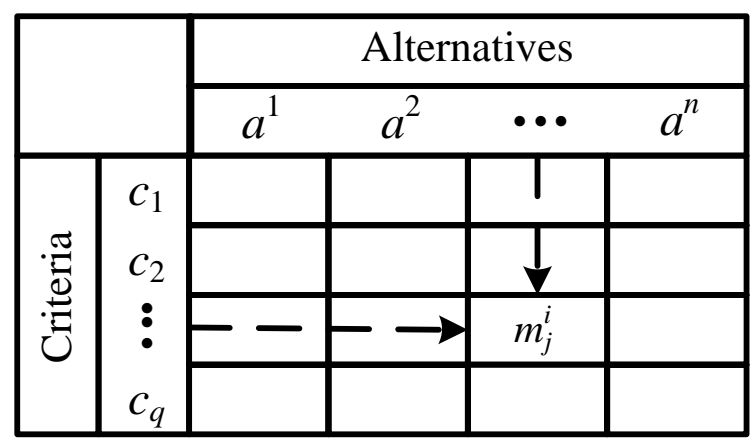

Fig. 1. Performance Matrix in MCDA, adapted from [8]

A DM's preferences are a significant determinant to solve any MCDA problem. Preferences can be expressed on consequences, called values, and preferences on criteria, referred to as weights [8]. Values, preferences on consequences, are obtained by processing consequences to reflect the DM's needs or objectives. For instance, a DM's preference on the reduction or increase of cost may be linear or non-linear. Generally, the relationship between consequences and values can be expressed as $v_{j}\left(a^{i}\right)=f_{j}\left(m_{j}^{i}\right)$, where $v_{j}\left(a^{i}\right)$ and $m_{j}^{i}$ are a value and a consequence measurement, respectively; $f_{j}(\cdot)$ is a real-valued function that maps consequences to values. The DM's values over all criteria 
for alternative $a^{i}$ are expressed as a value vector, $\mathbf{v}\left(a^{i}\right)=\left(v_{1}\left(a^{i}\right), v_{2}\left(a^{i}\right), \ldots, v_{q}\left(a^{i}\right)\right)$. Many consequence normalization processes in current literature can be regarded as maps from consequences to values. Preferences on criteria are usually expressed as weights, indicating the relative importance of criteria. Let the weight for criterion $c_{j} \in \mathbf{C}$ be $w_{j} \in \mathbb{R}^{+}$. Generally, the DM's weight vector, $\mathbf{w}=\left(w_{1}, w_{2}, \ldots, w_{j}, \ldots, w_{q}\right)$, is normalized to 1 , i.e. $\sum_{j=1}^{q} w_{j}=1$

Criteria are often categorized into three groups according to a DM's preference directions along consequences: benefit and cost criteria are monotonic, and preferences increase as consequences increase for benefit criteria but opposite for cost criteria. For non-monotonic criteria, the DM specifies a non-extreme consequence as the most or least preferred and preferences decrease or increase gradually when consequences move away from the most preferred or the least preferred consequence. Note that, as values are refined consequence data reflecting the DM's preferences, they are always preference monotonic, i.e. the larger a value, the more preferred or the less preferred, while the first case is more commonly used.

In summary, the difference between consequence data and preference data (values or weights) can be outlined as below.

- Consequences are relatively objective and original raw data, while preference data are subjective refined data to reflect a DM's preferences.

- Data ranges of consequences may vary significantly: some can be very large, and others may be very small; preference data are normalized data to a certain range. For instance, weights are usually set to between 0 and 1.

- In terms of the preference direction, consequences can be either monotonic or nonmonotonic while preferences are always monotonic.

After the problem is structured and preferences are obtained on consequences and an aggregation model is needed to generate an overall assessment for each alternative, thereby solve the specified problématique. Mathematically, for $a^{i} \in \mathbf{A}$, the evaluation of alternative $a^{i}, V\left(a^{i}\right)=F\left(\mathbf{v}\left(a^{i}\right), \mathbf{w}\right)$, is a real-valued mapping from the value vector $\mathbf{v}\left(a^{i}\right)$ and the weight vector $\mathbf{w}$ to a numerical evaluation of $a^{i}, V\left(a^{i}\right)$. A typical example is the linear additive value function, $V\left(a^{i}\right)=\sum_{j=1}^{q} w_{j} \cdot v_{j}\left(a^{i}\right)[17]$.

\subsection{The TOPSIS method}

The TOPSIS method is a distance-based approach, and its general procedure consists of the following steps [30]:

(1) Construct a performance matrix: An $n \times q$ matrix contains the raw consequence data for all alternatives against all criteria similar to Figure 1. 
(2) Normalize performance matrix: Apply a normalization process, $f_{j}(\cdot)$, to convert the original consequence data to values. For $\forall m_{j}^{i} \in \mathbb{R}^{+}$, three widely used normalization functions, mapping $m_{j}^{i}$ to $0 \leq v_{j}^{i} \leq 1$, are listed below [30]:

(a) Vector normalization: $v_{j}^{i}=\frac{m_{j}^{i}}{\sqrt{\sum_{i=1}^{n}\left(m_{j}^{i}\right)^{2}}}$;

(b) Sum-based linear normalization: $v_{j}^{i}=\frac{m_{j}^{i}}{\sum_{i=1}^{n}\left(m_{j}^{i}\right)}$.

(c) Min-Max-based normalization: $v_{j}^{i}=\frac{m_{j}^{i}}{\max _{i=1}^{n} m_{j}^{i}}\left(c_{j}\right.$ is a benefit criterion) and $v_{j}^{i}=\frac{\min _{i=1}^{n} m_{j}^{i}}{m_{j}^{i}}\left(c_{j}\right.$ is a cost criterion);

(3) Define the ideal and anti-ideal point: Set the ideal point, $a^{+}$, and anti-ideal point, $a^{-}$, based on the normalized performance matrix. For a benefit criterion, $c_{j}, v_{j}\left(a^{+}\right)=$ $\max _{i=1}^{n} v_{j}^{i}$ and $v_{j}\left(a^{-}\right)=\min _{i=1}^{n} v_{j}^{i}$; but for a cost criterion, $c_{k}, v_{j}\left(a^{+}\right)=\min _{i=1}^{n} v_{j}^{i}$ and $v_{j}\left(a^{-}\right)=\max _{i=1}^{n} v_{j}^{i}$.

(4) Assign weights to criteria: Set $w_{j}\left(w_{j} \in \mathbb{R}^{+}\right.$and $\left.\sum_{j=1}^{q} w_{j}=1\right)$ to represent the relative importance of criterion $c_{j}$.

(5) Calculate the distances of $a^{i}$ to the two ideal points, $a^{+}$and $a^{-}$: A commonly used distance definition is the $p$-norm distance function. Compute the distances of $a^{i}$ to $a^{+}$and $a^{-}$using $p$-norm distance functions, $D\left(a^{i}\right)^{+}=\left\{\sum_{j=1}^{q} w_{j} \mid\left(v_{j}\left(a^{+}\right)-\left.v_{j}\left(a^{i}\right)\right|^{p}\right\}^{1 / p}\right.$ and $D\left(a^{i}\right)^{-}=\left\{\sum_{j=1}^{q} w_{j} \mid\left(v_{j}\left(a^{i}\right)-\left.v_{j}\left(a^{-}\right)\right|^{p}\right\}^{1 / p}\right.$, where $p$ is a pre-defined distance norm, which is usually set as 1 or 2 and $|x|$ represents the absolute value of $x$.

(6) Obtain an integrated distance $a^{i}$ to these two extreme points: The distances of $a^{i}$ to the ideal and anti-ideal points have to be integrated to reach a final result. One way to integrate these two distances into an overall distance of $a^{i}, D\left(a^{i}\right)$, can be expressed as $D\left(a^{i}\right)=\frac{D\left(a^{i}\right)^{-}}{D\left(a^{i}\right)^{-}+D\left(a^{i}\right)^{+}}$, where a larger value of $D\left(a^{i}\right)$ represents a better overall performance.

\section{A DEA-TOPSIS method}

\section{$3.1 \quad$ Flexible settings of $a^{+}$and $a^{-}$}

As described in Section 2, the setting of ideal and anti-ideal points in the original TOPSIS is based upon value data that are normalized consequences reflecting the DM's preference directions over different criteria. $a^{+}$and $a^{-}$are set as the combinations of either maximum or minimum values of $v_{j}\left(a^{i}\right)\left(\forall c_{j} \in \mathbf{C}\right.$ and $\left.\forall a^{i} \in \mathbf{A}\right)$, depending on whether a criterion is benefit or cost. In practice, a DM may often have ideal or anti- 
ideal alternatives (points) directly on consequences, rather than on normalized values. For example, in business analysis, various benchmarks have been identified for company performance evaluations. To improve the flexibility in setting $a^{+}$and $a^{-}$, the approach reported in this article allows a DM to define $a^{+}$and $a^{-}$in the consequence space directly with the following conditions:

(1) $\forall a^{i} \in \mathbf{A}, D\left(a^{i}\right)^{+} \leq D\left(a^{-}\right)^{+}$: the normalized distance from $a^{-}$to $a^{+}$should be larger than that between any alternative $a^{i}$ in $\mathbf{A}$ and $a^{+}$;

(2) $\forall a^{i} \in \mathbf{A}, D\left(a^{i}\right)^{-} \leq D\left(a^{+}\right)^{-}$: the normalized distance from $a^{+}$to $a^{-}$should be larger than that between any alternative $a^{i}$ in $\mathbf{A}$ and $a^{-}$.

It is easy to verify that the setting of $a^{+}$and $a^{-}$in the original TOPSIS method satisfies these two conditions. Therefore, our new approach to setting $a^{+}$and $a^{-}$can be regarded as a natural extension of the original TOPSIS method.

To describe the distance definitions of different types of criteria more easily, let $\mathbf{C}=$ $\mathbf{C}^{c} \cup \mathbf{C}^{o}$, where $\mathbf{C}, \mathbf{C}^{c}$, and $\mathbf{C}^{o}$ represent the whole criteria set, quantitative (cardinal) criteria set and qualitative (ordinal) criteria set, respectively. Furthermore, let $\mathbf{C}^{c}=$ $\left\{c_{1}^{c}, \ldots, c_{j}^{c}, \ldots, c_{q_{c}}^{c}\right\}$ and $\mathbf{C}^{o}=\left\{c_{1}^{o}, \ldots, c_{j}^{o}, \ldots, c_{q_{o}}^{o}\right\}$, hence, $q=q_{c}+q_{o}$, where $q, q_{c}$ and $q_{o}$ are the size (cardinality) of $\mathbf{C}, \mathbf{C}^{c}$ and $\mathbf{C}^{o}$, respectively. Now, the detailed distance definitions for $\mathbf{C}^{c}$ and $\mathbf{C}^{o}$ are given below.

\subsection{Distance definitions and aggregation over $\mathbf{C}^{c}$}

Let $m_{j}^{c}\left(a^{i}\right)$ be the consequence measurement of $a^{i}$ on a quantitative criterion, $c_{j}^{c}$. When $a^{i}=a^{+}$or $a^{-}, m_{j}^{c}\left(a^{i}\right)=m_{j}^{c}\left(a^{+}\right)$or $m_{j}^{c}\left(a^{-}\right)$. For each $c_{j}^{c} \in \mathbf{C}^{c}$, the distances from $a^{i}$ to the predefined extreme points, $a^{+}$and $a^{-}$, are denoted as $\left|m_{j}^{c}\left(a^{+}\right)-m_{j}^{c}\left(a^{i}\right)\right|$ and $\left|m_{j}^{c}\left(a^{-}\right)-m_{j}^{c}\left(a^{i}\right)\right|$, respectively. Then, an appropriate normalization function in Section 2.2 can be chosen to obtain the normalized distances of $a^{i}$ to $a^{+}$and $a^{-}$, denoted by $d_{j}^{c}\left(a^{i}\right)^{+}$and $d_{j}^{c}\left(a^{i}\right)^{-}$, respectively, as detailed below. Note that in order to validate the two conditions in Section 3.1, the distance between $a^{+}$and $a^{-},\left|m_{j}^{c}\left(a^{+}\right)-m_{j}^{c}\left(a^{-}\right)\right|$, is included in the following normalization process. As a unique feature of the new distance definitions, the following normalization functions can be applied to any kind of criterion, benefit, cost, or non-monotonic. A DM does not need to explicitly differentiate these three types of criteria during a normalization process.

- Vector-based normalization:

First, set $\varepsilon_{j}^{+}=\sqrt{\sum_{i=1}^{n}\left(m_{j}^{c}\left(a^{+}\right)-m_{j}^{c}\left(a^{i}\right)\right)^{2}+\left(\left(m_{j}^{c}\left(a^{+}\right)-m_{j}^{c}\left(a^{-}\right)\right)^{2}\right.}$ as the ideal normalization factor, and $\varepsilon_{j}^{-}=\sqrt{\sum_{i=1}^{n}\left(m_{j}^{c}\left(a^{-}\right)-m_{j}^{c}\left(a^{i}\right)\right)^{2}+\left(\left(m_{j}^{c}\left(a^{-}\right)-m_{j}^{c}\left(a^{+}\right)\right)^{2}\right.}$ as the anti-ideal normalization factor.

Then, the normalized distance between $a^{i} \in \mathbf{A}$ and $a^{+}$over criterion $c_{j}$ is defined 
as: $d_{j}^{c}\left(a^{i}\right)^{+}=\frac{\left|m_{j}^{c}\left(a^{+}\right)-m_{j}^{c}\left(a^{i}\right)\right|}{\varepsilon_{j}^{+}}$, and the normalized distance between $a^{i} \in \mathbf{A}$ and $a^{-}$over criterion $c_{j}$ is $d_{j}^{c}\left(a^{i}\right)^{-}=\frac{\left|m_{j}^{c}\left(a^{-}\right)-m_{j}^{c}\left(a^{i}\right)\right|}{\varepsilon_{j}^{-}}$. By plugging $a^{-}$in $d_{j}^{c}(\cdot)^{+}$and $a^{+}$ $\operatorname{in} d_{j}^{c}(\cdot)^{-}, d_{j}^{c}\left(a^{-}\right)^{+}=\frac{\left|m_{j}^{c}\left(a^{+}\right)-m_{j}^{c}\left(a^{-}\right)\right|}{\varepsilon_{j}^{+}}$, and $d_{j}^{c}\left(a^{+}\right)^{-}=\frac{\left|m_{j}^{c}\left(a^{-}\right)-m_{j}^{c}\left(a^{+}\right)\right|}{\varepsilon_{j}^{-}}$.

- Sum-based absolute normalization:

Let $\varepsilon_{j}^{+}=\sum_{i=1}^{n}\left|m_{j}^{c}\left(a^{+}\right)-m_{j}^{c}\left(a^{i}\right)\right|+\mid\left(m_{j}^{c}\left(a^{+}\right)-m_{j}^{c}\left(a^{-}\right) \mid\right.$and $\varepsilon_{j}^{-}=\sum_{i=1}^{n}\left|m_{j}^{c}\left(a^{-}\right)-m_{j}^{c}\left(a^{i}\right)\right|+\mid\left(m_{j}^{c}\left(a^{-}\right)-m_{j}^{c}\left(a^{+}\right) \mid\right.$.

The two normalized distances are defined as $d_{j}^{c}\left(a^{i}\right)^{+}=\frac{\left|m_{j}^{c}\left(a^{+}\right)-m_{j}^{c}\left(a^{i}\right)\right|}{\varepsilon_{j}^{+}}$and $d_{j}^{c}\left(a^{i}\right)^{-}=\frac{\left|m_{j}^{c}\left(a^{-}\right)-m_{j}^{c}\left(a^{i}\right)\right|}{\varepsilon_{j}^{-}}$, respectively. Similarly, the two extreme distances are obtained as $d_{j}^{c}\left(a^{-}\right)^{+}=\frac{\left|m_{j}^{c}\left(a^{+}\right)-m_{j}^{c}\left(a^{-}\right)\right|}{\varepsilon_{j}^{+}}$, and $d_{j}^{c}\left(a^{+}\right)^{-}=\frac{\left|m_{j}^{c}\left(a^{-}\right)-m_{j}^{c}\left(a^{+}\right)\right|}{\varepsilon_{j}^{-}}$,

- Max-based absolute normalization:

First, set $\varepsilon_{j}^{+}=\max \left\{\max _{i=1}^{n}\left|m_{j}^{c}\left(a^{+}\right)-m_{j}^{c}\left(a^{i}\right)\right|,\left|m_{j}^{c}\left(a^{+}\right)-m_{j}^{c}\left(a^{-}\right)\right|\right\}$and $\varepsilon_{j}^{-}=$ $\max \left\{\max _{i=1}^{n}\left|m_{j}^{c}\left(a^{-}\right)-m_{j}^{c}\left(a^{i}\right)\right|,\left|m_{j}^{c}\left(a^{+}\right)-m_{j}^{c}\left(a^{-}\right)\right|\right\}$.

We define the two normalized distances as $d_{j}^{c}\left(a^{i}\right)^{+}=\frac{\left|m_{j}^{c}\left(a^{+}\right)-m_{j}^{c}\left(a^{i}\right)\right|}{\varepsilon_{j}^{+}}$and $d_{j}^{c}\left(a^{i}\right)^{-}=$ $\frac{\left|m_{j}^{c}\left(a^{-}\right)-m_{j}^{c}\left(a^{i}\right)\right|}{\varepsilon_{j}^{-}}$, and their two extreme cases are derived as $d_{j}^{c}\left(a^{-}\right)^{+}=\frac{\left|m_{j}^{c}\left(a^{+}\right)-m_{j}^{c}\left(a^{-}\right)\right|}{\varepsilon_{j}^{+}}$and $d_{j}^{c}\left(a^{+}\right)^{-}=\frac{\left|m_{j}^{c}\left(a^{-}\right)-m_{j}^{c}\left(a^{+}\right)\right|}{\varepsilon_{j}^{-}}$

One can verify that all the aforementioned normalized distances are between 0 and 1 , i.e. $\forall a^{i} \in \mathbf{A}$ and $\forall c_{j}^{c} \in \mathbf{C}^{c}, 0 \leq\left\{d_{j}^{c}\left(a^{i}\right)^{+}, d_{j}^{c}\left(a^{-}\right)^{+}\right\} \leq 1$ and $0 \leq\left\{d_{j}^{c}\left(a^{i}\right)^{-}, d_{j}^{c}\left(a^{+}\right)^{-}\right\} \leq 1$.

\subsection{Distance definitions and aggregation over $\mathbf{C}^{o}$}

As mentioned in Section 2, linguistic grade evaluations are commonly employed for measuring consequences over qualitative criteria, $\mathbf{C}^{o}$. Formally, let $\mathbf{L}=\left\{l_{1}, \ldots, l_{r}, \ldots, l_{m}\right\}$ as the linguistic grade set, where $l_{1}$ represents the best grade, $l_{2}$ the next best, $\cdots$, $L_{m}$, the worst grade. Then, $m_{j}^{o}\left(a^{i}\right)=l_{r}$ means that $a^{i}$ has been assessed at grade $l_{r}$ on criterion $c_{j}^{o}$, i.e. as the $r^{\text {th }}$ grade. For example, $m_{1}^{o}\left(a^{3}\right)=l_{2}$ means that alternative $a^{3}$ is considered to be the $2^{\text {nd }}$ best grade on ordinal criterion $c_{1}^{o}$. (For simplicity, we assume that all ordinal criteria are assessed on the same linguistic grade set.)

Since the linguistic grade set represents a preference order, obviously, $m_{j}^{o}\left(a^{+}\right)=l_{1}$ and $m_{j}^{o}\left(a^{-}\right)=l_{m}$, indicating that the linguistic grade assessment for $a^{+}$on criterion $c_{j}^{o}$ should be the best one, $l_{1}$, and the assessment of $a^{-}$should be the worst one, $l_{m}$. Let $d_{j}^{o}\left(a^{i}\right)^{+}$ 
and $d_{j}^{o}\left(a^{i}\right)^{-}$represent the distance between $a^{i}$ and $a^{+}$, and between $a^{i}$ and $a^{-}$over the criterion $c_{j}^{o}$, respectively. Similar to qualitative criterion case where distances are normalized to between 0 and 1, we have assumed that the distance between $a^{+}$and $a^{-}$ over $c_{j}^{o}$ is 1 , i.e., $d_{j}^{o}\left(a^{-}\right)^{+}=d_{j}^{o}\left(a^{+}\right)^{-}=1$. By piecewise linear interpolation, if $m_{j}^{o}\left(a^{i}\right)=$ $l_{r}$, then $d_{j}^{o}\left(a^{i}\right)^{+}$satisfies the condition, $\frac{r-1}{m} \leq d_{j}^{o}\left(a^{i}\right)^{+} \leq \frac{r}{m}$, and $d_{j}^{o}\left(a^{i}\right)^{-}$satisfies the condition, $\frac{m-r+1}{m} \leq d_{j}^{o}\left(a^{i}\right)^{-} \leq \frac{m-r}{m}$. Of course, additional qualitative criterion quantification methods such as the approaches in [9] can be employed to provide more sophisticated decision analysis.

After determining the normalized distances from each $a^{i}$ to $a^{+}$and $a^{-}$, an aggregated distance related to the so-called $p$-norm, where $p \geq 1$, is used to integrate the normalized distances, $d_{j}^{+}\left(a^{i}\right)$ and $d_{j}^{-}\left(a^{i}\right)$, over all criteria. The most widely adopted norms are $p=1$ and $p=2$.

Let $\mathbf{w}^{c}=\left(w_{1}^{c}, \ldots, w_{j}^{c}, \ldots, w_{q_{c}}^{c}\right)$ represent the weight information for $\mathbf{C}^{c}$ and $\mathbf{w}^{o}=$ $\left(w_{1}^{o}, \ldots, w_{j}^{o}, \ldots, w_{q_{o}}^{o}\right)$ denote the weight information for $\mathbf{C}^{o}$. Then, the weighted $p$-power distance of $a^{i}$ to $a^{+}$over $\mathbf{C}^{c}$ and $\mathbf{C}^{o}$ is $D\left(a^{i}\right)^{+}=\left\{\sum_{j=1}^{q_{c}} w_{j}^{c} \cdot\left(d_{j}^{c}\left(a^{i}\right)^{+}\right)^{p}+\sum_{j=1}^{q_{o}} w_{j}^{o} \cdot\left(d_{j}^{o}\left(a^{i}\right)^{+}\right)^{p}\right\}^{1 / p}$, and $D\left(a^{i}\right)^{-}=\left\{\sum_{j=1}^{q_{c}} w_{j}^{c} \cdot\left(d_{j}^{c}\left(a^{i}\right)^{-}\right)^{p}+\sum_{j=1}^{q_{o}} w_{j}^{o} \cdot\left(d_{j}^{o}\left(a^{i}\right)^{-}\right)^{p}\right\}^{1 / p}$.

Obviously, when $a^{i}=a^{-}, D\left(a^{i}\right)^{+}=D\left(a^{-}\right)^{+}$, and $D\left(a^{i}\right)^{-}=D\left(a^{+}\right)^{-}$for $a^{i}=a^{+}$.

\subsection{DEA-based optimization models}

The parameters, $\mathbf{w}^{c}, \mathbf{w}^{o}, d_{j}^{o}\left(a^{i}\right)^{+}$and $d_{j}^{o}\left(a^{i}\right)^{-}, \forall c_{j}^{o} \in \mathbf{C}^{o}$ and $a^{i} \in \mathbf{A}$, need to be specified before a TOPSIS analysis is implemented. Also, the two conditions of setting $a^{+}$and $a^{-}$in Section 3.1 have to be verified. Here, two DEA-based optimization models are designed to tackle these tasks simultaneously.

\subsubsection{Individual optimization models}

Two individual optimization models are designed to identify the required information from both $a^{+}$and $a^{-}$points of view.

$\mathbf{P}\left(a^{i}\right)^{+}$

Minimize: $D\left(a^{i}\right)^{+}$

\section{Subject to:}


$\forall a^{i} \in \mathbf{A}, D\left(a^{i}\right)^{+} \leq D\left(a^{-}\right)^{+} \leq 1$

$\forall a^{i} \in \mathbf{A}$, if $m_{j}^{o}\left(a^{i}\right)=l_{r}$, then $\frac{r-1}{m} \leq d_{j}^{o}\left(a^{i}\right)^{+} \leq \frac{r}{m}$;

$\forall c_{j}^{o} \in \mathbf{C}^{o}, d_{j}^{o}\left(a^{-}\right)^{+}=1$

$\sum_{j=1}^{q_{c}} w_{j}^{c}+\sum_{j=1}^{q_{o}} w_{j}^{o}=1$

$\forall c_{j}^{c} \in \mathbf{C}^{c}, w_{j}^{c} \geq \rho$ and $\forall c_{j}^{o} \in \mathbf{C}^{o}, w_{j}^{o} \geq \rho$.

$\mathbf{P}\left(a^{i}\right)^{-}$

Maximize: $D\left(a^{i}\right)^{-}$

Subject to:

$\forall a^{i} \in \mathbf{A}, D\left(a^{i}\right)^{-} \leq D\left(a^{+}\right)^{-} \leq 1 ;$

$\forall a^{i} \in \mathbf{A}$, if $m_{j}^{o}\left(a^{i}\right)=l_{r}$, then $\frac{m-r+1}{m} \leq d_{j}^{o}\left(a^{i}\right)^{-} \leq \frac{m-r}{m} ;$

$\forall c_{j}^{o} \in \mathbf{C}^{o}, d_{j}^{o}\left(a^{+}\right)^{-}=1$

$\sum_{j=1}^{q_{c}} w_{j}^{c}+\sum_{j=1}^{q_{o}} w_{j}^{o}=1$

$\forall c_{j}^{c} \in \mathbf{C}^{c}, w_{j}^{c} \geq \rho$ and $\forall c_{j}^{o} \in \mathbf{C}^{o}, w_{j}^{o} \geq \rho$.

Note that $\rho \in \mathbb{R}^{+}$is a pre-defined small positive value to ensure all weights are positive. For example, if all calculations are rounded to four decimal places, then $\rho$ could be set as 0.0001 or any meaningful value greater than 0.0001 .

Following the final aggregation procedure in the TOPSIS method, the overall distance performance of $a^{i}, D\left(a^{i}\right)$, can be calculated using $D\left(a^{i}\right)=\frac{D\left(a^{i}\right)^{-}}{D\left(a^{i}\right)^{-}+D\left(a^{i}\right)^{+}}$.

\subsubsection{An integrated optimization model}

Next, an optimization model integrating the two distance measurements from both $a^{+}$ and $a^{-}$is designed to identify the required information.

$\mathbf{P}\left(a^{i}\right)$

Maximize: $\frac{D\left(a^{i}\right)^{-}}{D\left(a^{i}\right)^{-}+D\left(a^{i}\right)^{+}}$ 


\section{Subject to:}

$$
\begin{aligned}
& \forall a^{i} \in \mathbf{A}, D\left(a^{i}\right)^{+} \leq D\left(a^{-}\right)^{+} \leq 1 ; \\
& \forall a^{i} \in \mathbf{A}, D\left(a^{i}\right)^{-} \leq D\left(a^{+}\right)^{-} \leq 1 ; \\
& \forall a^{i} \in \mathbf{A}, \text { if } m_{j}^{o}\left(a^{i}\right)=l_{r}, \text { then } \frac{r-1}{m} \leq d_{j}^{o}\left(a^{i}\right)^{+} \leq \frac{r}{m} \text { and } \frac{m-r+1}{m} \leq d_{j}^{o}\left(a^{i}\right)^{-} \leq \frac{m-r}{m} ; \\
& \forall c_{j}^{o} \in \mathbf{C}^{o}, d_{j}^{o}\left(a^{-}\right)^{+}=1 ; \\
& \forall c_{j}^{o} \in \mathbf{C}^{o}, d_{j}^{o}\left(a^{+}\right)^{-}=1 ; \\
& \sum_{j=1}^{q_{c}} w_{j}^{c}+\sum_{j=1}^{q_{o}} w_{j}^{o}=1 ; \\
& \forall c_{j}^{c} \in \mathbf{C}^{c}, w_{j}^{c} \geq \rho \text { and } \forall c_{j}^{o} \in \mathbf{C}^{o}, w_{j}^{o} \geq \rho .
\end{aligned}
$$

Note that $\mathbf{P}\left(a^{i}\right)^{+}$provides the estimate of weight and qualitative criterion quantification from the ideal point perspective by minimizing the distance from $a^{i}$ to $a^{+}$, and $\mathbf{P}\left(a^{i}\right)^{-}$ furnishes similar information from the anti-ideal point perspective by maximizing the distance from $a^{i}$ to $a^{-}$, while $\mathbf{P}\left(a^{i}\right)$ is designed to integrate the two analysis procedures into an aggregated assessment.

Since the constraints in $\mathbf{P}\left(a^{i}\right)^{+}, \mathbf{P}\left(a^{i}\right)^{-}$and $\mathbf{P}\left(a^{i}\right)$ define a closed and bounded set. The objective functions are continuous functions on these sets. Therefore, the extreme value theorem of advanced calculus [16] implies that the programs attain minimum or maximum values at least once. Lingo [21] is comprehensive commercial software designed to solve linear, nonlinear and integer optimization models faster, easier and more efficient and ${ }_{\perp}$ it is employed for the calculation in the case study.

Both models follow the general philosophy of DEA to identify individual alternatives' best possible overall performance from both the ideal and anti-ideal points. Since the information of weights and qualitative criterion quantification is calculated by $\mathbf{P}\left(a^{i}\right)^{+}$ and $\mathbf{P}\left(a^{i}\right)^{-}$in two separate optimization models, differences may exist. In the meantime, the integrated optimization model is designed to synthesize the two perspectives from $a^{+}$and $a^{-}$and provide an overall assessment of the relevant information, furnishing a middling and, presumably, better choice for employment. Next, a case study adapted from [30], in which Shih et al. extended the TOPSIS method for group decision making, is used to demonstrate the proposed method. 


\section{A numerical example}

\subsection{Background information}

This example is adapted from Shih et al. [30]. A local chemical company is recruiting an on-site manager. 17 qualified candidates (treated as alternatives) are short-listed (labelled as $a^{1}-a^{17}$ ). The human resources department provides some standard selection tests, consisting of three knowledge tests: 1. language test, 2. professional test and 3. safety rule test as well as two skill tests: 4. professional skills and 5. computer skills. In addition, two interviews are administered: 6. panel interview and 7. 1-on-1 interview. Each candidate will be assessed on the seven criteria constituting the criterion set $\mathbf{C}$. Obviously, the first five criteria are generally measured on a quantitative and objective basis, while the last two criteria may be conveniently evaluated in a subjective manner. Here, the first five criteria are treated as quantitative criteria, $\mathbf{C}^{c}$, and labelled as $c_{1}^{c}-c_{5}^{c}$ sequentially, and the last two criteria are regarded as qualitative criteria, $\mathbf{C}^{o}$, and labelled as $c_{1}^{o}$ and $c_{2}^{o}$.

Instead of directly assigning quantitative values for $c_{1}^{o}$ and $c_{2}^{o}$ as [30], a linguistic grade set, $\mathbf{L}=\left\{l_{1}, l_{2}, l_{3}, l_{4}\right\}$, representing four ordinal assessments, is employed to characterize the DM's evaluation. Here we take the first DM's quantitative assessments regarding $c_{1}^{o}$ and $c_{2}^{o}$ in [30] as the basis (as a group decision making problem, there are four DMs in [30]) and apply the following transformation rules: if $90 \leq m_{j}\left(a^{i}\right) \leq 100$, then $a^{i}$ belongs to $l_{1}$ on criterion $c_{j}$; if $80 \leq m_{j}\left(a^{i}\right)<90$, then $a^{i}$ belongs to $l_{2}$; if $70 \leq m_{j}\left(a^{i}\right)<80$, then $a^{i}$ belongs to $l_{3}$; and if $m_{j}\left(a^{i}\right)<70$, then $a^{i}$ belongs to $l_{4}$.

It is further assumed that all quantitative criteria are benefit. Following the ideal and anti-ideal points setting in [30] (Note Shih et al.'s paper [30] did not provide $a^{+}$and $a^{-}$in consequence data format explicitly, but this information can be easily traced under monotonic assumptions.), $\forall c_{j}^{c} \in \mathbf{C}^{c}, m_{j}^{c}\left(a^{+}\right)=\max _{i=1}^{17} m_{j}^{c}\left(a^{i}\right)$ and $m_{j}^{c}\left(a^{-}\right)=$ $\min _{i=1}^{17} m_{j}^{c}\left(a^{i}\right)$. The basic consequence information of these 17 alternatives, $a^{+}$and $a^{-}$ over the seven criteria is listed in Table 1.

\subsection{A DEA-based optimization analysis}

It is assumed that all calculations are rounded to four decimal places, and $\rho=0.0001$. Furthermore, let $p=1$ and the vector-based normalization explained in Section 3.2 is used to conduct the analysis. The normalized distances from $a^{i} \in \mathbf{A}$ to $a^{+}$and $a^{-}$are listed in Tables 2 and 3, respectively.

Then, $\mathbf{P}\left(a^{i}\right)^{+}$and $\mathbf{P}\left(a^{i}\right)^{-}$are employed to calculate the optimal results of $D\left(a^{i}\right)^{+}$and $D\left(a^{i}\right)^{-}$, respectively. Subsequently, the generated optimal results of $D\left(a^{i}\right)^{+}$and $D\left(a^{i}\right)^{-}$ 
Table 1

Basic consequence information

\begin{tabular}{l|lllllll}
\hline \multirow{2}{*}{ Alternative } & \multicolumn{7}{|c}{ Criteria } \\
& $c_{1}^{c}$ & $c_{2}^{c}$ & $c_{3}^{c}$ & $c_{4}^{c}$ & $c_{5}^{c}$ & $c_{1}^{o}$ & $c_{2}^{o}$ \\
\hline$a^{1}$ & 80 & 70 & 87 & 77 & 76 & $l_{2}$ & $l_{3}$ \\
$a^{2}$ & 85 & 65 & 76 & 80 & 75 & $l_{4}$ & $l_{3}$ \\
$a^{3}$ & 78 & 90 & 72 & 80 & 85 & $l_{1}$ & $l_{2}$ \\
$a^{4}$ & 75 & 84 & 69 & 85 & 65 & $l_{4}$ & $l_{3}$ \\
$a^{5}$ & 84 & 67 & 60 & 75 & 85 & $l_{3}$ & $l_{2}$ \\
$a^{6}$ & 85 & 78 & 82 & 81 & 79 & $l_{2}$ & $l_{2}$ \\
$a^{7}$ & 77 & 83 & 74 & 70 & 71 & $l_{4}$ & $l_{3}$ \\
$a^{8}$ & 78 & 82 & 72 & 80 & 78 & $l_{3}$ & $l_{4}$ \\
$a^{9}$ & 85 & 90 & 80 & 88 & 90 & $l_{2}$ & $l_{2}$ \\
$a^{10}$ & 89 & 75 & 79 & 67 & 77 & $l_{3}$ & $l_{3}$ \\
$a^{11}$ & 65 & 55 & 68 & 62 & 70 & $l_{4}$ & $l_{4}$ \\
$a^{12}$ & 70 & 64 & 65 & 65 & 60 & $l_{4}$ & $l_{4}$ \\
$a^{13}$ & 95 & 80 & 70 & 75 & 70 & $l_{3}$ & $l_{3}$ \\
$a^{14}$ & 70 & 80 & 79 & 80 & 85 & $l_{2}$ & $l_{3}$ \\
$a^{15}$ & 60 & 78 & 87 & 70 & 66 & $l_{4}$ & $l_{4}$ \\
$a^{16}$ & 92 & 85 & 88 & 90 & 85 & $l_{1}$ & $l_{1}$ \\
$a^{17}$ & 86 & 87 & 80 & 70 & 72 & $l_{2}$ & $l_{2}$ \\
\hline$a^{+}$ & 95 & 90 & 88 & 90 & 90 & $l_{1}$ & $l_{1}$ \\
$a^{-}$ & 60 & 55 & 60 & 62 & 60 & $l_{4}$ & $l_{4}$ \\
\hline
\end{tabular}

for each alternative are aggregated as per $D\left(a^{i}\right)=\frac{D\left(a^{i}\right)^{-}}{D\left(a^{i}\right)^{-}+D\left(a^{i}\right)^{+}}$to produce the final distance performance. The relevant distances and their associated rankings are given in Table 4 under the heading "Individual Models".

Next, the integrated optimization model in Section 3.4.2 is employed to produce the overall distance performance for each alternative directly. The detailed calculation is omitted here and the final result of distances and rankings are shown in Table 4 under the "Integrated Model" heading.

A close examination of Table 4 reveals that the two DEA-based methods, both the individual optimization models and the integrated optimization model, provide roughly consistent rankings. For example, only the ranking orders between $10^{\text {th }}-12^{\text {th }}$ are different and other rankings are almost identical. It is also noticed that ties tend to appear in the individual optimization models, while the integrated model seems to have more discrimination power to break ties between alternatives. 
Table 2

Normalized distance information to $a^{+}$

\begin{tabular}{|c|c|c|c|c|c|c|c|}
\hline \multirow{2}{*}{ Alternative } & \multicolumn{7}{|c|}{ Criteria } \\
\hline & $c_{1}^{c}$ & $c_{2}^{c}$ & $c_{3}^{c}$ & $c_{4}^{c}$ & $c_{5}^{c}$ & $c_{1}^{o}$ & $c_{2}^{o}$ \\
\hline$a^{1}$ & 0.1836 & 0.2678 & 0.0151 & 0.1829 & 0.1898 & {$[0.25,0.5]$} & {$[0.5,0.75]$} \\
\hline$a^{2}$ & 0.1224 & 0.3348 & 0.1817 & 0.1407 & 0.2034 & {$[0.75,1]$} & {$[0.5,0.75]$} \\
\hline$a^{3}$ & 0.2081 & 0.0000 & 0.2423 & 0.1407 & 0.0678 & {$[0,0.25]$} & {$[0.25,0.5]$} \\
\hline$a^{4}$ & 0.2448 & 0.0804 & 0.2877 & 0.0704 & 0.3389 & {$[0.75,1]$} & {$[0.5,0.75]$} \\
\hline$a^{5}$ & 0.1346 & 0.3080 & 0.4240 & 0.2111 & 0.0678 & {$[0.5,0.75]$} & {$[0.25,0.5]$} \\
\hline$a^{6}$ & 0.1224 & 0.1607 & 0.0908 & 0.1266 & 0.1491 & {$[0.25,0.5]$} & {$[0.25,0.5]$} \\
\hline$a^{7}$ & 0.2203 & 0.0937 & 0.2120 & 0.2814 & 0.2576 & {$[0.75,1]$} & {$[0.5,0.75]$} \\
\hline$a^{8}$ & 0.2081 & 0.1071 & 0.2423 & 0.1407 & 0.1627 & {$[0.5,0.75]$} & {$[0.75,1]$} \\
\hline$a^{9}$ & 0.1224 & 0.0000 & 0.1211 & 0.0281 & 0.0000 & {$[0.25,0.5]$} & {$[0.25,0.5]$} \\
\hline$a^{10}$ & 0.0734 & 0.2009 & 0.1363 & 0.3236 & 0.1762 & {$[0.5,0.75]$} & {$[0.5,0.75]$} \\
\hline$a^{11}$ & 0.3672 & 0.4687 & 0.3028 & 0.3940 & 0.2711 & {$[0.75,1]$} & {$[0.75,1]$} \\
\hline$a^{12}$ & 0.3060 & 0.3482 & 0.3482 & 0.3518 & 0.4067 & {$[0.75,1]$} & {$[0.75,1]$} \\
\hline$a^{13}$ & 0.0000 & 0.1339 & 0.2725 & 0.2111 & 0.2711 & {$[0.5,0.75]$} & {$[0.5,0.75]$} \\
\hline$a^{14}$ & 0.3060 & 0.1339 & 0.1363 & 0.1407 & 0.0678 & {$[0.25,0.5]$} & {$[0.5,0.75]$} \\
\hline$a^{15}$ & 0.4284 & 0.1607 & 0.0151 & 0.2814 & 0.3254 & {$[0.75,1]$} & {$[0.75,1]$} \\
\hline$a^{16}$ & 0.0367 & 0.0670 & 0.0000 & 0.0000 & 0.0678 & {$[0,0.25]$} & {$[0,0.25]$} \\
\hline$a^{17}$ & 0.1102 & 0.0402 & 0.1211 & 0.2814 & 0.2440 & {$[0.25,0.5]$} & {$[0.25,0.5]$} \\
\hline$a^{-}$ & 0.4284 & 0.4687 & 0.4240 & 0.3940 & 0.4067 & 1 & 1 \\
\hline
\end{tabular}

\section{Conclusions}

A hybrid approach of integrating DEA into TOPSIS is designed to capitalize on the unique features from both methods for improving multicriteria decision analysis. A numerical example is developed to demonstrate the proposed method and a comparative study is carried out. The results illustrate the consistency of ranking results from both individual and integrated optimization models, implying potential applicability of the proposed approach.

\section{References}

[1] M.A. Abo-Sinna, A.H. Amer, Extensions of TOPSIS for multi-objective large-scale nonlinear programming problems. Applied Mathematics and Computation 162(1) (2005) 243-256.

[2] M.A. Abo-Sinna, A.H. Amerb, A.S. Ibrahim, Extensions of TOPSIS for large scale multi-objective non-linear programming problems with block angular structure, Applied Mathematical Modelling 32(3) (2008) 292-302. 
Table 3

Normalized distance information to $a^{-}$

\begin{tabular}{l|ccccccc}
\hline \multirow{2}{*}{ Alternative } & \multicolumn{7}{|c}{ Criteria } \\
& $c_{1}^{c}$ & $c_{2}^{c}$ & $c_{3}^{c}$ & $c_{4}^{c}$ & $c_{5}^{c}$ & $c_{1}^{o}$ & $c_{2}^{o}$ \\
\hline$a^{1}$ & 0.2082 & 0.1417 & 0.3474 & 0.2076 & 0.2019 & {$[0.5,0.75]$} & {$[0.25,0.5]$} \\
$a^{2}$ & 0.2602 & 0.0944 & 0.2058 & 0.2492 & 0.1893 & {$[0,0.25]$} & {$[0.25,0.5]$} \\
$a^{3}$ & 0.1874 & 0.3306 & 0.1544 & 0.2492 & 0.3154 & {$[0.75,1]$} & {$[0.5,0.75]$} \\
$a^{4}$ & 0.1561 & 0.2739 & 0.1158 & 0.3184 & 0.0631 & {$[0,0.25]$} & {$[0.25,0.5]$} \\
$a^{5}$ & 0.2498 & 0.1133 & 0.0000 & 0.1799 & 0.3154 & {$[0.25,0.5]$} & {$[0.5,0.75]$} \\
$a^{6}$ & 0.2602 & 0.2172 & 0.2830 & 0.2630 & 0.2397 & {$[0.5,0.75]$} & {$[0.5,0.75]$} \\
$a^{7}$ & 0.1770 & 0.2644 & 0.1801 & 0.1107 & 0.1388 & {$[0,0.25]$} & {$[0.25,0.5]$} \\
$a^{8}$ & 0.1874 & 0.2550 & 0.1544 & 0.2492 & 0.2271 & {$[0.25,0.5]$} & {$[0,0.25]$} \\
$a^{9}$ & 0.2602 & 0.3306 & 0.2573 & 0.3599 & 0.3785 & {$[0.5,0.75]$} & {$[0.5,0.75]$} \\
$a^{10}$ & 0.3019 & 0.1889 & 0.2444 & 0.0692 & 0.2145 & {$[0.25,0.5]$} & {$[0.25,0.5]$} \\
$a^{11}$ & 0.0520 & 0.0000 & 0.1029 & 0.0000 & 0.1262 & {$[0,0.25]$} & {$[0,0.25]$} \\
$a^{12}$ & 0.1041 & 0.0850 & 0.0643 & 0.0415 & 0.0000 & {$[0,0.25]$} & {$[0,0.25]$} \\
$a^{13}$ & 0.3643 & 0.2361 & 0.1286 & 0.1799 & 0.1262 & {$[0.25,0.5]$} & {$[0.25,0.5]$} \\
$a^{14}$ & 0.1041 & 0.2361 & 0.2444 & 0.2492 & 0.3154 & {$[0.5,0.75]$} & {$[0.25,0.5]$} \\
$a^{15}$ & 0.0000 & 0.2172 & 0.3474 & 0.1107 & 0.0757 & {$[0,0.25]$} & {$[0,0.25]$} \\
$a^{16}$ & 0.3331 & 0.2833 & 0.3602 & 0.3876 & 0.3154 & {$[0.75,1]$} & {$[0.75,1]$} \\
$a^{17}$ & 0.2706 & 0.3022 & 0.2573 & 0.1107 & 0.1514 & {$[0.5,0.75]$} & {$[0.5,0.75]$} \\
\hline$a^{+}$ & 0.3643 & 0.3306 & 0.3602 & 0.3876 & 0.3785 & 1 & 1 \\
\hline
\end{tabular}

[3] A. Charnes, W.W. Cooper, E. Rhodes E., Measuring Efficiency of Decision-Making Units, European Journal of Operational Research, 2 (1978) 428-449.

[4] C.T. Chen, Extensions of the TOPSIS for group decision-making under environment, Fuzzy Sets and Systems 114 (2000) 1-9.

[5] M.F. Chen, G.H. Tzeng, Combining gray relation and TOPSIS concepts for selecting an expatriate host country, Mathematical and Computer Modelling 40 (2004) 1473-1490.

[6] Y. Chen, K.W. Li, D.M. Kilgour, K.W. Hipel, A Case-based distance model for multiple criteria ABC analysis, Computers and Operations Research, 35 (2008) 776-796.

[7] Y. Chen, X. Su, K.W. Hipel, An Index Aggregation Approach to Comparing the Overall Performance of Emerging and Developed Countries, Socio-Economic Planning Sciences, 43 (2009) 25-39.

[8] Chen, Y., Kilgour, D.M., Hipel, K.W.: Multiple criteria classification with an application in water resources planning, Computers and Operations Research, 33 (2006) 3301-3323.

[9] W. Cook, M. Kress, Ordinal Information and Preference Structures: Decision Models and Applications, Prentice-Hall, Englewood Cliffs, NJ, 1991.

[10] W. Cook, M. Kress, A Multiple-criterion composite index model for quantitative and qualitative data. European Journal of Operational Research 78 (1994) 367-379. 
Table 4

Final distance performance and rankings

\begin{tabular}{c|cc|cc|cc|cc|}
\hline \multirow{2}{*}{ Alternative } & \multicolumn{7}{|c|}{ Individual Models } & \multicolumn{2}{|c}{ Integrated Model } \\
& $D\left(a^{i}\right)^{+}$ & Ranking1 & \multicolumn{2}{c}{$D\left(a^{i}\right)^{-}$} & Ranking2 & Aggregation & Ranking3 & \multicolumn{2}{c}{$D\left(a^{i}\right)$} & Ranking4 \\
\hline$a^{1}$ & 0.0152 & 5 & 0.7497 & $(5-8)$ & 0.9801 & 5 & 0.9580 & 5 \\
$a^{2}$ & 0.1225 & 15 & 0.4998 & $(11-14)$ & 0.8031 & 15 & 0.6798 & 15 \\
$a^{3}$ & 0.0001 & 3 & 0.9996 & 2 & 0.9999 & $(2-3)$ & 0.9999 & 2 \\
$a^{4}$ & 0.0706 & 10 & 0.4998 & $(11-14)$ & 0.8763 & 11 & 0.8185 & 10 \\
$a^{5}$ & 0.0679 & 9 & 0.7497 & $(6-8)$ & 0.9169 & $(8-9)$ & 0.8228 & 9 \\
$a^{6}$ & 0.0909 & 12 & 0.7498 & $(3-4)$ & 0.8919 & 10 & 0.7570 & 12 \\
$a^{7}$ & 0.0939 & 13 & 0.4998 & $(11-14)$ & 0.8419 & 13 & 0.7380 & 13 \\
$a^{8}$ & 0.1072 & 14 & 0.4998 & $(11-14)$ & 0.8234 & 14 & 0.7040 & 14 \\
$a^{9}$ & 0.0001 & 2 & 0.7498 & $(3-4)$ & 0.9999 & $(2-3)$ & 0.9998 & 3 \\
$a^{10}$ & 0.0735 & 11 & 0.4999 & $(9-10)$ & 0.8717 & 12 & 0.8041 & 11 \\
$a^{11}$ & 0.2712 & 16 & 0.2499 & $(16-17)$ & 0.4795 & 16 & 0.3175 & 16 \\
$a^{12}$ & 0.3061 & 17 & 0.2499 & $(16-17)$ & 0.4495 & 17 & 0.2538 & 17 \\
$a^{13}$ & 0.0002 & 4 & 0.4999 & $(9-10)$ & 0.9996 & 4 & 0.9995 & 4 \\
$a^{14}$ & 0.0679 & 8 & 0.7497 & $(6-8)$ & 0.9169 & $(8-9)$ & 0.8229 & 8 \\
$a^{15}$ & 0.0154 & 6 & 0.3473 & 15 & 0.9576 & 6 & 0.9576 & 6 \\
$a^{16}$ & 0.0000 & 1 & 0.9997 & 1 & 1.0000 & 1 & 1.0000 & 1 \\
$a^{17}$ & 0.0403 & 7 & 0.7497 & $(5-8)$ & 0.9490 & 7 & 0.8823 & 7 \\
\hline Note that “( & $(x-y) "$ & represents that the ranking & of an alternative ties between $x$ and $y$.
\end{tabular}

[11] Department of the Environment, Transport and the Regions (DETR), UK, A New Deal for Trunk Roads in England: Understanding the New Approach to Appraisal, London, UK, 1998.

[12] J. Doyle, R. Green, Efficiency and cross-efficiency in DEA: derivations, meanings and uses. Operational Research Society 45 (1994) 567-578.

[13] J. Doyle, Multiattribute choice for the lazy decision maker: let the alternatives decide!. Organizational Behavior and Human Decision Processes 62 (1995) 87-100.

[14] A. Emrouznejad, B.R. Parker and G. Tavares, Evaluation of research in efficiency and productivity: a survey and analysis of the first 30 years of scholarly literature in DEA, Socio-Economic Planning Sciences, in Press, available online on December 7, 2007.

[15] J. Figueira, S. Greco, M. Ehrgott, (Eds.) Multiple Criteria Decision Analysis: State of the Art Surveys, Springer, New York, 2005.

[16] P.M. Fitzpatrick, Advanced Calculus: A Course in Mathematical Analysis, Thomson Brooks-Cole, Boston, 1995.

[17] C.L. Hwang, K. Yoon, Multiple Attribute Decision Making, Springer-Verlag, Berlin, 1981.

[18] G. Islei, A.G. Lockett, Judgemental modelling based on geometric least square, European Journal of Operational Research 36 (1988) 27-35.

[19] Y.J. Lai, T.Y. Liu and C.L. Hwang, TOPSIS for MODM, European Journal of Operational Research 76 (1994) 486-500. 
[20] M.J. Liberatore, R.L. Nydick, The analytic hierarchy process in medical and health care decision making: A literature review, European Journal of Operational Research 189 (2008), Pages 194-207.

[21] LINDO Systems. Inc. Lingo software. http://www.lindo.com, cited on December 5, 2005.

[22] W.L. Ng, A simple classifier for multiple criteria ABC analysis, European Journal of Operational Research 177 (2007) 344-353.

[23] R.L. Keeney and H. Raiffa, Decisions with Multiple Objectives: Preferences and Value Tradeoffs, New York: Wiley, 1976.

[24] G. Kim, C.S. Park and K.P. Yoon, Identifying investment opportunities for advanced manufacturing systems with comparative-integrated performance measurement, International Journal of Production Economics 50 (1997) 23-33.

[25] R. Ramanathan, Data envelopment analysis for weight derivation and aggregation in the analytic hierarchy process, Computers and Operations Research 33 (2006) 1289-1307.

[26] B. Roy, Multicriteria Methodology for Decision Aiding, Kluwer, Dordrecht, 1996.

[27] T.L. Saaty, Analytic Hierarchy Process, McGraw Hill New York, 1980.

[28] T. Sexton, R. Silkman, A. Hogan, Data envelopment analysis: critique and extensions. In: Silkman R, editor. Measuring efficiency: an assessment of data envelopment analysis. San Francisco: Jossey-Bass, 1986.

[29] J. Seydel, Data envelopment analysis for decision support, Industrial Management \& Data Systems 106 (2006) 81-95.

[30] H.S. Shih, H.J. Shyur and E.S. Lee, An extension of TOPSIS for group decision making, Mathematical and Computer Modelling 45 (2007) 801-813.

[31] H.S. Shih, Incremental analysis for MCDM with an application to group TOPSIS, European Journal of Operational Research 186 (2008) 720-734.

[32] E.A. Silver, D.F. Pyke and R. Peterson, Inventory Management and Production Planning and Scheduling, 3rd edition, Wiley, New York, 1998.

[33] T. Stewart, Relationships between data envelopment analysis and multicriteria decision analysis, Journal of the Operational Research Society 47 (1996) 654-665.

[34] Y.M. Wang, K.S. Chin, A new data envelopment analysis method for priority determination and group decision making in the analytic hierarchy process, European Journal of Operational Research 195 (2009) 239-250.

[35] D. von Winterfeldt, W. Edwards, Decision Analysis and Befuwioral Research, Cambridge University Press, New York, 1986. 